\title{
Application Study on Multiple Network Communication of Power Engineering
}

\author{
Zhang LiMing \\ Kunming university of science and engineering economy \\ and manages an academy, Kunming, China, 650093 \\ grabc@qq.com
}

\author{
Guo Rui \\ Kunming University of Science and Technology, \\ Kunming,China, 650051 \\ Zhang5115804@163.com
}

\begin{abstract}
The application of network communication in the field of engineering monitoring software has been very common, but most of them used a network communication mode. This paper introduces the multiple network communication modes (asynchronous network socket communication, wave communication, named pipe communication and mail slot communication) to communicate according to the specific requirements of the project, and each of communication mode features is well applied to engineering practice to meet the requirements of engineering, so as to improve the efficiency of the communication.
\end{abstract}

Key words-network socket; network communication; multiwave; named pipe

\section{INTRODUCTION}

This paper describes the data network engineering using power quality data collected by LabView software in power system, all the communication computers to connected in star way to the same LAN, the engineering requires to transmit the real-time data collected by LabView software in lower computers, and processing by communication server after the data forwarding to communication server, and part of the data store in SQL SERVER database (for WEB publishing server providing data); part of the data forwarding to local users again for the local users online real-time view, at the same time, the command of local user to lower computers also needs through communication server forwarding. Engineering data network model as shown in figure 1 below.

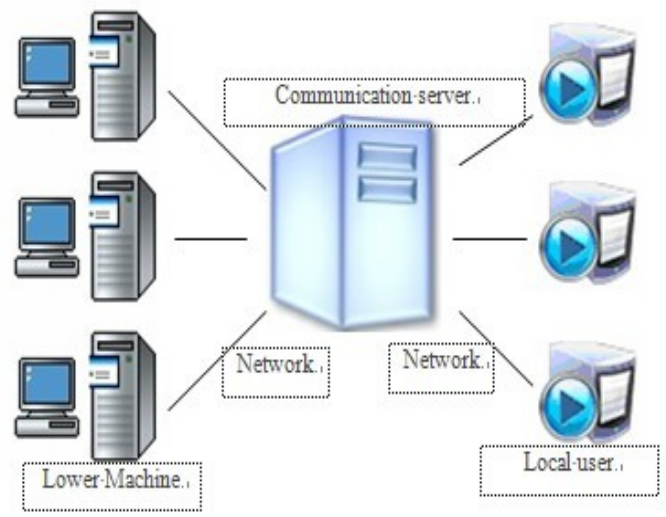

Figure 1 Communication model
Lower computer: used for collecting the data and active upload to communication server.

Communication server: used for forwarding the data and command of lower computer and far user.

Far user: providing a good human-machine interface for operators to check the relevant data.

\section{OPERATING ENVIRONMENT}

- Operating system: Windows 2000

- Compile environment: $\mathrm{vc}++6.0$

\section{SYSTEM EXPLANATION}

The system ${ }^{[1]}$ may have more than one local users and lower computer systems, the numbers of lower computer systems are fixed in general after design but the numbers of local users may change at any moment. Any computers in local area network shall become local users as long as the installation and operation of local client software, and when local client software stop running from the computers, the computers is no longer the local users. Communication server in the system is the only, used for forwarding data.

Communication of system is based on the network, thus in the network we want to control data throughput to appropriate use of bandwidth. The numbers of local users are changing from time to time so in the system may have any number of local users, and every local user can view any site equipment information. Lower computers transmit data only in the local user requirements, so at ordinary times, only part of lower computers need to transmit information, if we usually will all of the data of lower computers online transmission will affect the efficiency of transmission, will take up too much bandwidth. So we need to control lower computers to transmit data only when the user needs to transmit data. When local users check information of lower computers, the best situation is all users checking information from only one device, so we just need to send the data of this device, the worst is different users to view different lower computers data, which will lead to all the lower computers are required to transmit data, and one a lower computer may receive a lot of requests from local users. A reference counter in the data acquisition and sending software of lower computers is used to record the numbers of requested users. When the counter is zero show that no user requests data from the device, and thus the 
device will stop sending data to the client, or send data to the client.

\section{DESIGN PROCESS}

In the system we need to design three kinds of software: the data acquisition and sending software of lower computers, communication server software of communication server, and local user software of local client. Data flow in the system is analyzed first:

\section{A Data declaration} kinds:

The transmissions of data in the system have three

1) Real-time data: data collected by lower computers system software and sending to communication server. The lower computers system data acquisition and sending software decide whether to send data according to the reference count, if send, passes the data to the communication server, and communication server forward the data to local users.

Features: the mass data of continuous transmission.

2) Alarm data: record fault information data generated when a fault occurs.

Features: data transmission from time to time.

3) Command data: data the local users sending to the lower computers system.

First the data are sent to communication server, and then to data acquisition and sending software of the lower computers system by communication server, and the software to execute the orders of the local users.Features: small batch data transmission from time to time.

\section{B Data transmit instructions}

The communication application model is designed according to the type of data flow, different data by different transmission channel and the specific requirements of the engineering.Network communication application model is shown in Figure 2.

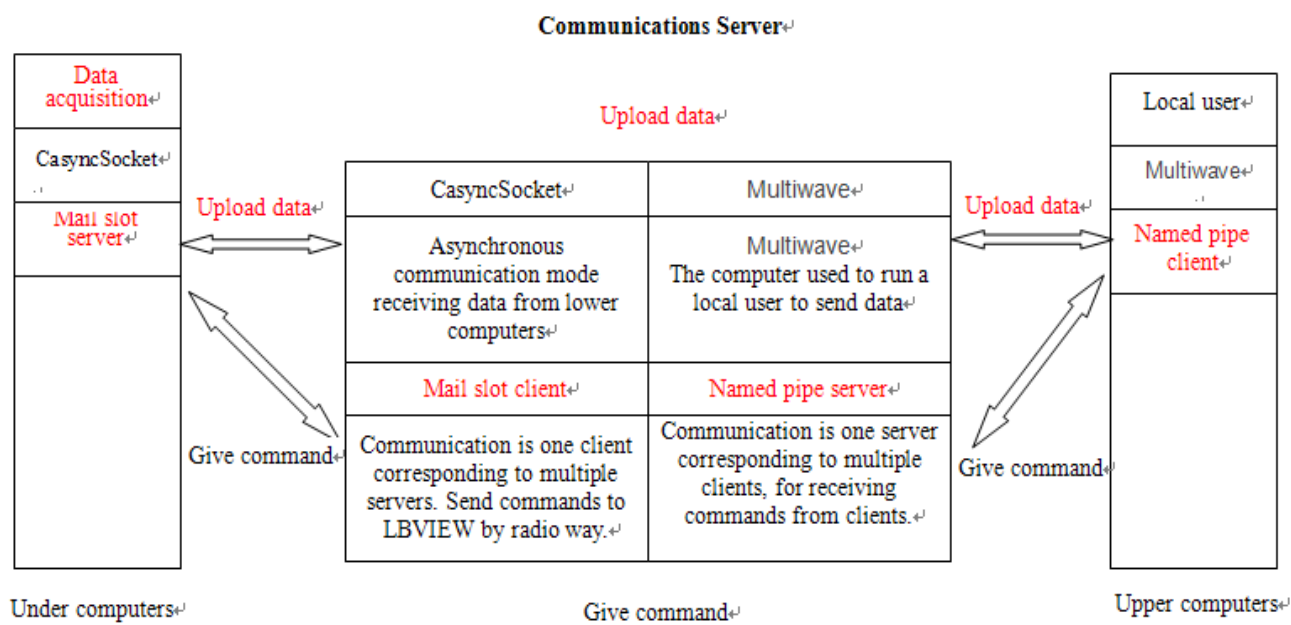

Figure 2 Communication application model

The characteristics of each network communication mode ${ }^{[2,3]}$ and the applications in this system are analyzed as the following:

Mail slot technology: mail slot is characteristic of the single-phase communications only, that is, transmit data from the client to the server and the client can transmit data to more than one server at the same time. Communicating in UDP way, the length of data can't more than 424 bytes, so this way is appropriate for transmitting a small amount data from point to more.

This system installed the mail slot client in communication server, and installed the mail slot server software in data acquisition and sending software. Communication server will send orders given by client to the mail slot server in the data acquisition and sending software through the mail slot client. Because there may be many lower computers, so mail slot client can send the command broadcast to the mail slot server of every lower computer in the data acquisition and sending software, the lower computers change the value of the reference count by analysis of the order.

Named pipe technology: in two-way reliable data transmission (via TCP/IP mode), but communication only between the server and client, and a server can receive more client data, this accords with the requirements of one communication server receiving multiple user command data, so in communication server creating named pipe server, and in local client creating named pipe client. So every client is a named pipe client, every client can send command data to the named pipe server in communication server.

Multiwave communication technology: a kind of deformation of radio communication mode. Radio mode send broadcast data to all machines in local area network, so a lot of occupation of the network resources; Wave communication mode only receives data from the same 
wave group member, and the machines not in the wave group can't receive the data sent by wave group members. So we can well control the broadcast storm and able to send batch data to many computers, compared to mail slot which can only send a small amount of data. So we add wave communication code in communication server and the software of each local client, communication server forward the data receiving from data acquisition and sending software by wave mode to other wave users (local users). As long as in the local area network to add the local user is equivalent to adding the user to the wave group, so the wave data sent by communication server can be received by local users, which meet with our demands too.

CAsyncSocket communication: refers to asynchronous network socket communication, used for transmission data between lower computer and communication server. In communication server, is running the asynchronous network socket server program, the program receives the data from multiple clients, and the lower computers use the client program.

\section{CONCLUSIONS}

This paper mainly introduces a programming methods combined with engineering practice of how to use of multiple network communication modes to transmit data, provides a communication model. So in the future if a similar project required, the same method can be applied, and the concrete implementation details may be different depending on the engineering.

\section{REFERENCES}

[1] Zhou Deze, Yuan Naner, Computer intelligent monitoring control system design and application, Beijing: Tsinghua university press, 2002

[2] Yang Xinqiang, Data communication and computer network, Beijing: Electronic industry press, 2003

[3] Lang Rui, Luo Fagen, Visual c ++ networking communication program development guide, Beijing: Mechanical industry press, 2004

[4] Wang Hairui, Remote monitoring system based on MC9S12NE64 and Z-WAVE technology, ICEEE2010 EI: 20110313605087 